05

\title{
Нелинейная динамика деформационных полос в алюминий-магниевом сплаве при испытании на ползучесть
}

\author{
(C) А.А. Шибков, М.А. Желтов, М.Ф. Гасанов, А.Е. Золотов \\ Тамбовский государственный университет им. Г.Р. Державина, \\ 392000 Тамбов, Россия \\ e-mail: shibkov@tsu.tmb.ru
}

(Поступило в Редакцию 31 января 2017 г.)

\begin{abstract}
Проведены экспериментальные исследования различных типов пластических неустойчивостей в ходе прерывистой ползучести на примере алюминий-магниевого сплава АМг6. Показано, что прерывистая ползучесть проявляет пороговую динамику: деформационная ступень на кривой ползучести амплитудой $~ 1-6 \%$ стартует, когда скорость предшествующей непрерывной ползучести достигает некоторого критического значения. В ходе развития ступени скорость деформации меняется в интервале более двух порядков и происходят переходы между различными динамическими режимами типов $A$ и $B$, которые характеризуются разными уровнями регулярности скачков напряжения в силовом отклике. Обсуждается нелинейные аспекты деформационного поведения сплава в условиях прерывистой ползучести.
\end{abstract}

DOI: 10.21883/JTF.2017.10.44996.2185

\section{Введение}

Прерывистая деформация вызывает в последнее время интерес как пример сложности пространственновременной динамики, возникающей вследствие коллективного поведения дислокаций [1-3]. Вместе с тем неустойчивое течение является технологически важной проблемой, поскольку оно неблагоприятно воздействует на формуемость промышленных металлических сплавов. В частности, связанная с пластической неустойчивостью локализация деформации в полосах ухудшает механические свойства конструкционного материала и может вызвать его преждевременную коррозию и внезапное разрушение. Кроме того, статические или распространяющиеся полосы деформации портят качество поверхности промышленных изделий.

Большинство исследований прерывистого пластического течения было выполнено с постоянной скоростью деформации в жесткой машине на растяжение, когда оно проявляет себя скачками разгрузки - эффект Портевена-Ле Шателье (ПЛШ) [1-8]. Значительно меньше исследований использовали условия постоянной скорости роста напряжения в мягкой машине на растяжение [4-6], несмотря на то что ранние наблюдения ступенчатой пластической деформации в этих условиях восходят к первой половине XIX столетия. Лишь очень небольшое внимание было уделено исследованиям прерывистой ползучести [7-10] по сравнению с эффектом ПЛШ в металлических сплавах. Прерывистая ползучесть проявляет себя в виде деформационных ступеней амплитудой $\sim 1-6 \%$ на первой и второй стадиях ползучести и описывалась в литературе как „внезапное удлинение“, „спонтанная деформация“ или „деформационный взрыв“. В противоположность случаю постоянной скорости деформации и постоянной (ненулевой) скорости роста напряжения данные о ступенчатом отклике на кривых ползучести (лестничная ползучесть) крайне ограничены.

В настоящее время проблема деформационных макроступеней и природа пространственно-временных картин деформационных полос в ходе прерывистой ползучести остается слабо понятой из-за сравнительно небольшого количества работ и практического отсутствия таких исследований в последние три десятилетия. В то же время изучение механизмов прерывистой ползучести и полосообразования имеет большое практическое значение. Кроме того, с позиции нелинейной динамики важно понимать механизм спонтанного формирования пространственно-временных (диссипативных) структур макролокализованной пластической деформации в условиях заданной (контролируемой) внешней силы, т.е. в динамически определенной системе.

Цель работы - экспериментально исследовать in situ критические условия формирования макроскопического деформационного скачка в условиях прерывистой ползучести алюминий-магниевого сплава АМг6, корреляции между силовым откликом на развитие скачка деформации и пространственно-временными структурами деформационных полос, а также исследовать переходы между различными динамическими режимами пластических неустойчивостей и проанализировать признаки динамического хаоса и самоорганизующейся критичности.

\section{1. Методика}

Материалом исследования является промышленный алюминий-магниевый сплав АМг6 (Al - 6.15\% Mg$0.65 \% \mathrm{Mn}-0.25 \% \mathrm{Si}-0.21 \% \mathrm{Fe}-0.1 \% \mathrm{Cu}-0.12 \%$ $\mathrm{Znwt. \% ),} \mathrm{проявляющий} \mathrm{скачкообразную} \mathrm{деформацию}$ в жесткой и мягкой испытательных машинах $[11,12]$, в том числе в условиях ползучести $[13,14]$. Плоские образцы в форме двусторонних лопаток размерами 
рабочей части $40 \times 3 \times 0.5 \mathrm{~mm}$ вырезались машинным способом из холоднокатаной полосы вдоль направления прокатки. Перед испытанием образцы отжигались при $450^{\circ} \mathrm{C}$ в течение $1 \mathrm{~h}$ и закаливались на воздухе. После термообработки средний размер зерна составил около $10 \mu \mathrm{m}$. Результаты исследования микроструктуры сплава представлены в работе [15]. Комплекс высокоскоростных методов регистрации деформации, нагрузки и исследования динамики и морфологии деформационных полос, a также схема растяжения в мягкой испытательной машине описаны в $[13,14]$.

\section{2. Результаты и обсуждение}

\section{1. Критическое условие возникновения деформационного скачка в ходе ползучести}

Образцы нагружались в две последовательные стадии: 1) стадии нагружения с постоянной скоростью $\dot{\sigma}_{0}=1.5 \mathrm{MPa} / \mathrm{s}$ до момента времени $t_{0}$, соответствующего приложенному напряжению $\sigma_{0}=(0.8-0.9) \sigma_{u}$ (рис. 1 , линия $0 A$ ), где $\sigma_{u} \approx 320 \mathrm{MPa}-$ предел прочности, и 2) стадии ползучести при инженерном напряжении $\sigma_{0}=$ const (рис. 1, линия $A B$ ). Спустя время $\tau$ по-

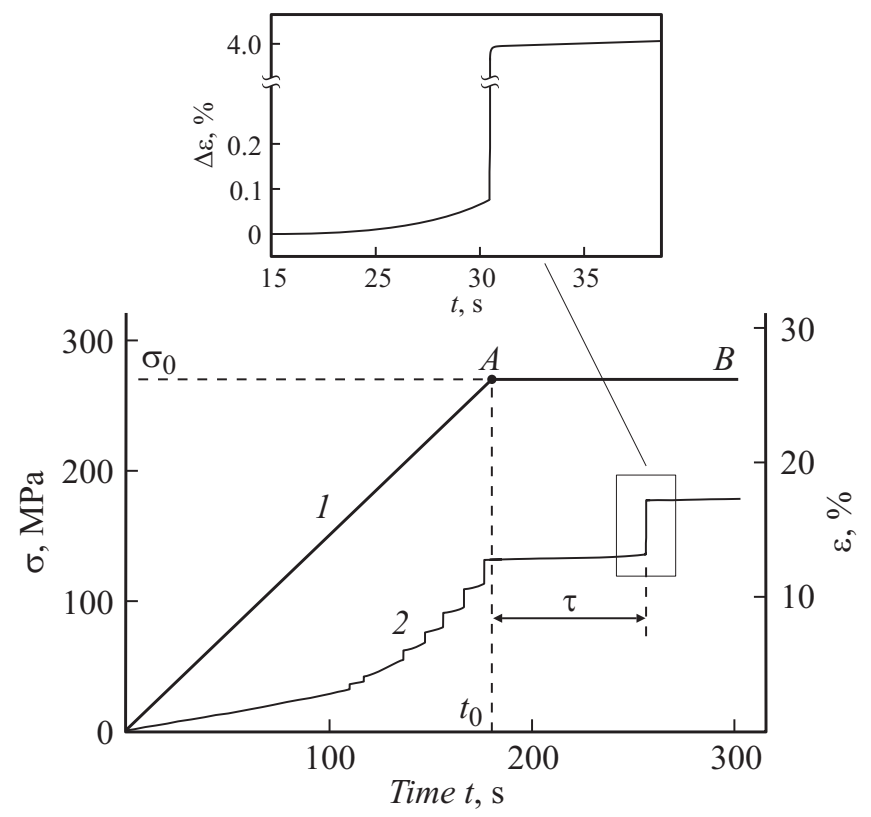

Рис. 1. Диаграмма нагружения образца в мягкой испытательной машине. 1 - зависимость от времени приложенного напряжения $\sigma(t): 0 A-$ стадия активного нагружения с постоянной скоростью возрастания напряжения $\dot{\sigma}_{0}=1.5 \mathrm{MPa} / \mathrm{s}$ до фиксированного напряжения $\sigma_{0}=270 \mathrm{MPa}$ при $t_{0}=180 \mathrm{~s}$; $A B$ - стадия ползучести, $\sigma_{0}=$ const. $2-$ временна́я зависимость относительной деформации $\varepsilon(t) . \tau-$ инкубационный интервал, т.е. время ожидания деформационного скачка в режиме ползучести. На вставке представлен скачок деформации амплитудой $\Delta \varepsilon_{m} \approx 4 \%$. До скачка скорость ползучести растет непрерывно аналогично третьей стадии ползучести. сле начала стадии ползучести образец теряет устойчивость, и сложная пространственно-временная структура деформационных полос спонтанно распространяется на поверхности образца вдоль направления растяжения. Следует подчеркнуть, что непосредственно перед стартом деформационного скачка скорость непрерывной ползучести возрастает по степенному закону $\dot{\varepsilon}=a \exp (b t)$ подобно скоростному поведению в ходе третьей стадии ползучести (см. вставку на рис. 1), где $a \approx 10^{-5} \mathrm{~s}^{-1}, b \approx 0.3 \mathrm{~s}^{-1}$ при приложенном напряжении $\sigma_{0}=270-280 \mathrm{MPa}$, которое значительно превышает условный предел текучести $\sigma_{0.2} \approx 155 \mathrm{MPa}$ и критическое напряжение появления первого деформационного скачка $\sigma_{c} \approx 165 \mathrm{MPa}$ при комнатной температуре и скорости нагружения $\dot{\sigma}_{0} \sim 1-3 \mathrm{MPa} / \mathrm{s}$. В отличие от „обычной“ третьей стадии непрерывной ползучести критическим макроскопическим событием является не разрыв образца, а деформационный скачок - крупная, амплитудой несколько процентов ступень на кривой ползучести.

Деформационный скачок стартует в момент времени, когда скорость непрерывной ползучести, как обнаружено, достигает некоторого критического значения $\dot{\varepsilon}_{\mathrm{cr} 1} \sim 10^{-4} \mathrm{~s}^{-1}$. Каждый последующий скачок деформации стартует после дополнительного нагружения образца напряжением 5-10 МРа, когда скорость предшествующей непрерывной ползучести достигает критического

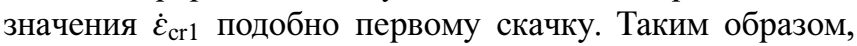
выявлено эмпирическое условие начала деформационного скачка в ходе непрерывной ползучести сплава АМг6 при комнатной температуре: до старта деформационного скачка скорость непрерывной ползучести меняется во времени от постоянной (подобно второй стадии ползучести) к степенной зависимости (подобно третьей стадии ползучести) до критического значения $\dot{\varepsilon}_{\mathrm{cr} 1} \sim 10^{-4} \mathrm{~s}^{-1}$. Типичная амплитуда деформационных скачков в этих условиях испытания, как обнаружено, равна $\sim 1-6 \%$, а время ожидания деформационного скачка („инкубационный“ интервал) $\tau \sim 10-100 \mathrm{~s}$.

\section{2. Корреляция между деформационным и силовым откликами и динамикой деформационных полос}

Данные видеосъемки со скоростью 500 frames/s показывают, что как и при нагружении с заданной скоростью $\dot{\sigma}_{0}=$ const [6], в условиях ползучести при $\sigma_{0}>\sigma_{0.2}$ деформационный скачок стартует одновременно с зарождением и последующим расширением первичной полосы деформации, которая представляет собой расширяющуюся шейку, наклоненную к оси образца под углом около $60^{\circ}$. В ходе расширения границы полосы движутся антипараллельно так, что „центр тяжести“ полосы остается неподвижным. Расширение полосы, как обнаружено, состоит из двух последовательных стадий:

1) первой стадии (стадии разгрузки) - очень быстрого расширения в течение $2-10 \mathrm{~ms}$ со скоростью границы 

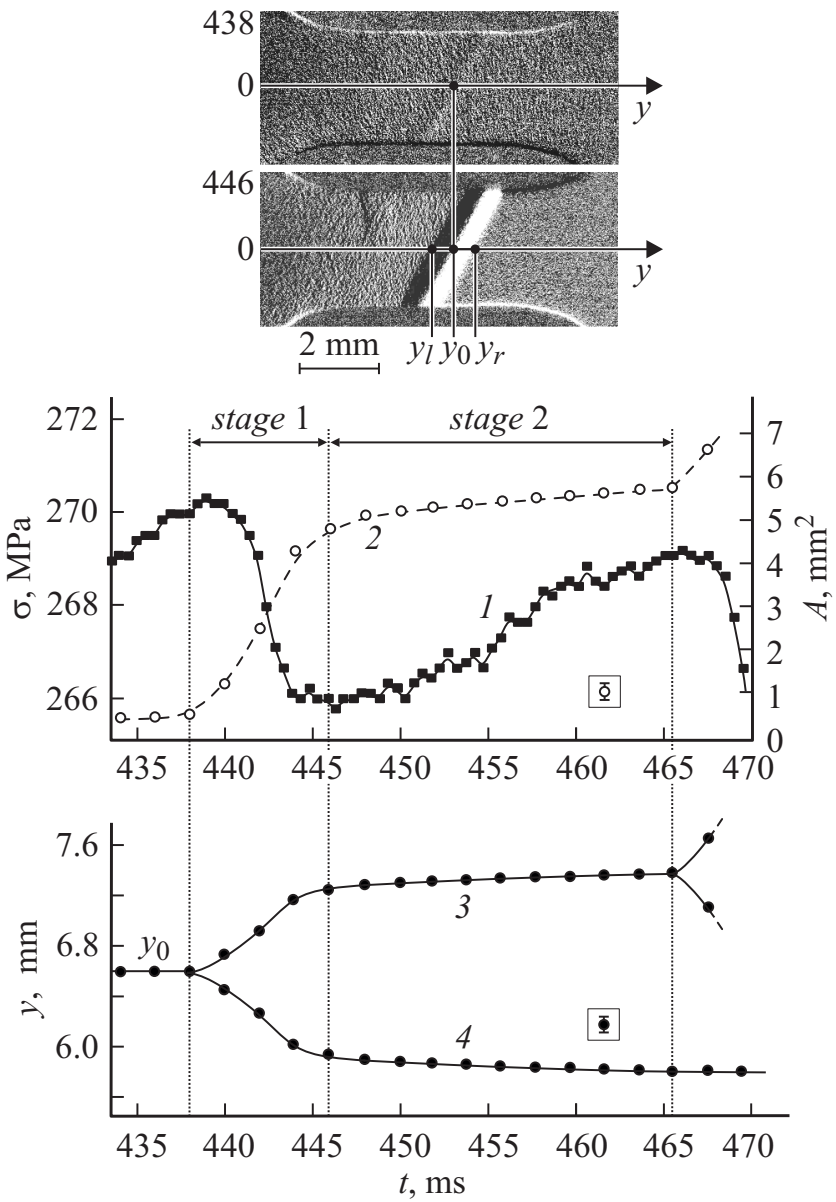

Рис. 2. Сравнение временных зависимостей напряжени $\sigma(1)$, площади $A(2)$ расширяющейся полосы деформации и координат правой $y_{r}(3)$ и левой $y_{l}(4)$ границ деформационной полосы. Горизонтальными стрелками отмечены быстрая и медленная стадии (стадии 1 и 2 соответственно) расширения полосы. На вставке показаны начальное и конечное изображения расширяющейся полосы на стадии 1 с позицией зарождения $\left(y_{0}\right)$ полосы и позициями правой $\left(y_{r}\right)$ и левой $\left(y_{l}\right)$ границ этой полосы соответственно.

несколько десятков cm/s, когда ширина полосы достигает более 90\% конечного значения (около $2 \mathrm{~mm}$ ); эта стадия сопровождается резким скачком разгрузки амплитудой $\sim 3-10 \mathrm{MPa}$;

2) второй стадии (стадии восстановления нагрузки) - медленного расширения в течение от нескольких десятков $\mathrm{ms}$ до нескольких сотен $\mathrm{ms}$ со средней скоростью границы около $3 \mathrm{~mm} / \mathrm{s}$; эта стадия сопровождается частичным или полным восстановлением напряжения.

На рис. 2 представлен типичный пример двустадийного расширения полосы деформации. Как видно, продолжительности первой и второй стадий расширения равны 8 и $20 \mathrm{~ms}$ соответственно. Кроме того, форма скачка напряжения на первой стадии хорошо коррелирует с временной зависимостью площади полосы $A(t)=\left[y_{r}(t)-y_{l}(t)\right] w(t)$, где $y_{r}(t)$ и $y_{l}(t)$ - временные зависимости координаты правой и левой границ полосы соответственно, $w(t)$ - ширина образца. На стадии восстановления нагрузки скорость границ полосы вдоль оси растяжения постепенно уменьшается, и когда она достигает нижнего предела ( $1 \mathrm{~mm} / \mathrm{s})$, границы полосы генерируют полосы в различные моменты времени, отстоящие на 100-200 ms. Вторичные полосы, как правило, идентичны „материнской“ полосе и также представляют собой расширяющиеся шейки, наклоненные под углом 55-63 к направлению растяжения. Затем границы вторичных полос генерируют полосы третьего поколения и т.д.

На рис. 3 представлены данные синхронной записи деформации $\Delta \varepsilon$ и силового отклика $\sigma(t)$ на развитие деформационной ступени амплитудой $\Delta \varepsilon_{m}$ около $4 \%$ на кривой ползучести (кривые 1 и 2 на рис. 3). Данные скоростной, со скоростью $2000 \mathrm{frames} / \mathrm{s}$, видеосъемки обработаны в виде так называемой корреляционной диаграммы - временной зависимости координат $y(t)$

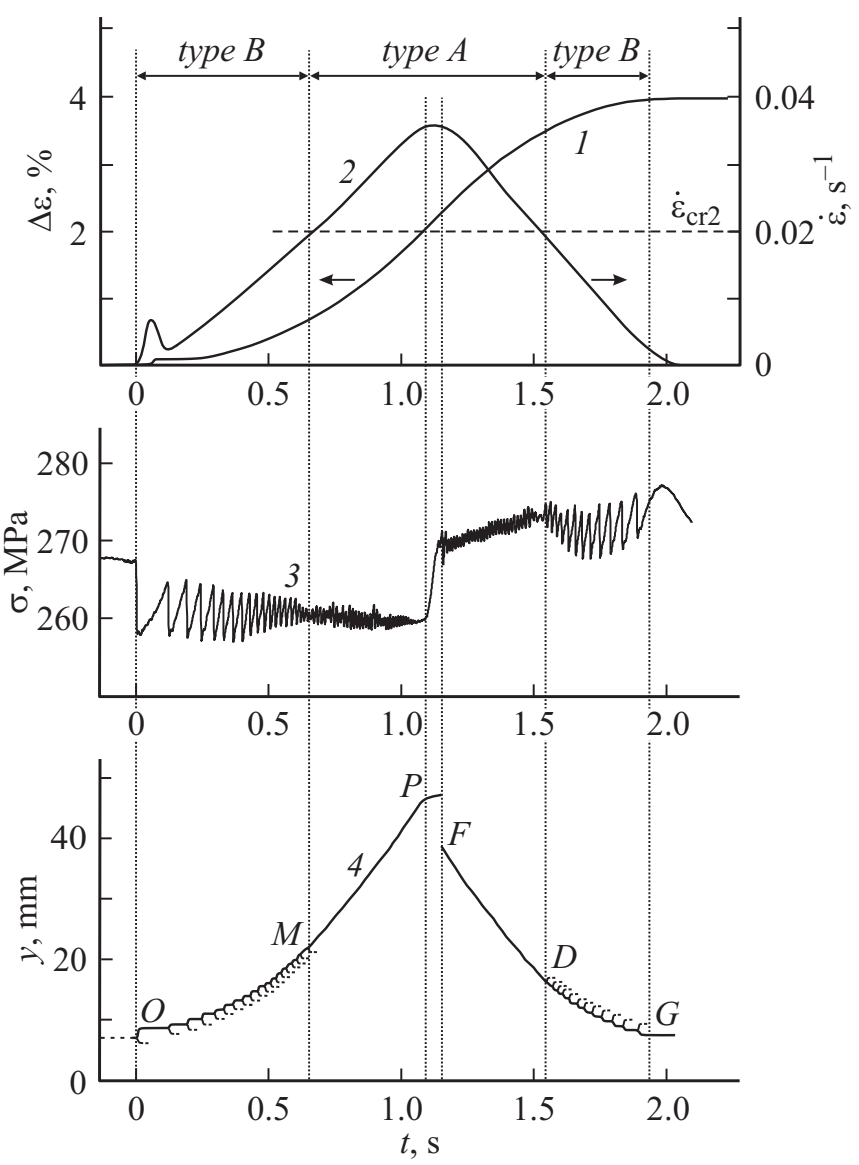

Рис. 3. Временные зависимости приращения деформации $\Delta \varepsilon$ (1) скорости деформации $\dot{\varepsilon}(2)$, развиваемой в ходе деформационного скачка, силового отклика $\sigma$ (3) и координаты $y$ (4) границы полос (корреляционная диаграмма) в образце сплава АМг6 при приложенном инженерном напряжении $\sigma_{0}=268 \mathrm{MPa}$. Вертикальные линии отмечают различные типы (A и $B)$ пластических неустойчивостей. Горизонтальная штриховая линия указывает вторую критическую деформацию $\dot{\varepsilon}_{\mathrm{cr} 2} \approx 2 \cdot 10^{-2} \mathrm{~s}^{-1}$, при которой происходит переход между динамическими режимами типов $A$ и $B$. 


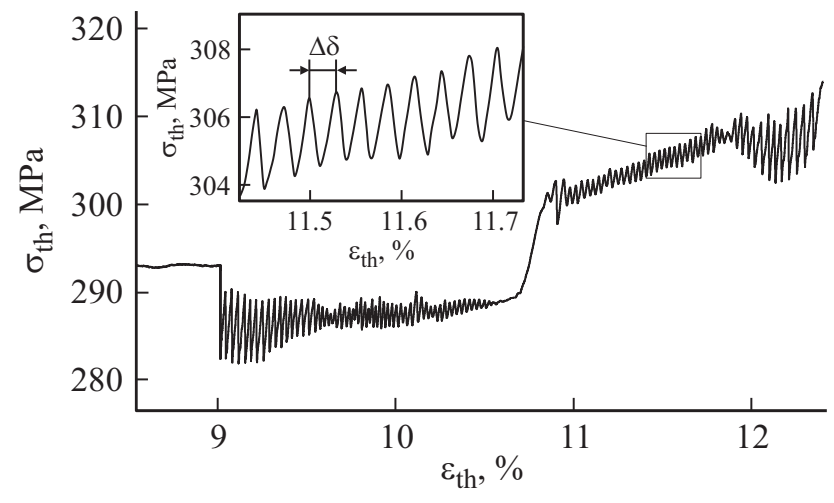

Pис. 4. Зависимость истинного напряжения $\sigma_{t h}$ от истинной деформации $\varepsilon_{t h}$, полученной из данных записи датчиков положения и усилия, представленных на рис. 3 (кривые 1 и 3 соответственно) в ходе развития деформационного скачка. На вставке показан фрагмент этой зависимости и отмечено приращение $\Delta \delta$ истинной деформации между соседними скачками истинного напряжения.

границ деформационных полос, которые зарождаются и расширяются в ходе развития деформационной ступени. Основные особенности этой корреляционной диаграммы описаны в работе [14]. В частности, установлено, что корреляционная диаграмма состоит из двух ветвей, разделенных точкой перегиба зависимости $\Delta \varepsilon(t)$, в которой скорость деформации максимальна $\dot{\varepsilon}_{m} \approx 4 \cdot 10^{-2} \mathrm{~s}^{-1}$. Данные видеосъемки показывают, что макролокализованная деформация зарождается около одной лопатки образца и распространяется сначала скачками подобно полосе ПЛШ типа $B$ за счет эстафетной передачи деформации от одной полосы (расширяющейся шейки) к другой по механизму, описанному выше, а затем после точки перегиба зависимости $\Delta \varepsilon(t)$ - квазинепрерывно аналогично полосе ПЛШ типа $A$, которая распространяется до противоположной лопатки образца, достигая ее в момент времени $P$ (см. кривую 4 на рис. 3). В последующий момент времени $F$ у этой лопатки зарождается новая полоса, которая движется в обратном направлении, демонстрируя противоположное деформационное поведение: сначала она распространяется непрерывно подобно полосе типа $A$, а затем после точки $D-$ дискретно скачками подобно полосе типа $B$.

Рассмотрим подробнее переходы между различными динамическими режимами в ходе развития индивидуальной ступени на кривой ползучести. Отметим, что мгновенная скорость деформации образца $\dot{\varepsilon}=\partial[\Delta \varepsilon(t)] / \partial t$, как обнаружено, меняется в широком диапазоне от $\dot{\varepsilon}_{\text {cr1 }} \sim 10^{-4} \mathrm{~s}^{-1}$ до $\dot{\varepsilon}_{m} \approx 10^{-2} \mathrm{~s}^{-1}$ в ходе эволюции одного деформационного скачка и происходят переходы между поведениями ПЛШ типов $B$ и $A$, а именно: пластическая неустойчивость типа $A$ наблюдается в скоростном интервале $2 \cdot 10^{-2}-4 \cdot 10^{-2} \mathrm{~s}^{-1}$. В то же время, если скорость деформации образца падает ниже значения $2 \cdot 10^{-2} \mathrm{~s}^{-1}$, то неустойчивость типа $A$ переходит в неустойчивость типа $B$.

Полученные результаты согласуются с условием переходов $B-A$ при испытаниях с постоянной скоростью деформирования $\dot{\varepsilon}_{0}=$ const в жестких испытательных машинах. Из литературных данных известно [16,17], что переход между различными режимами ПЛШ контролируется одним скалярным параметром: скоростью деформирования $\dot{\varepsilon}_{0}$, которая поддерживается постоянной в ходе испытания в жесткой машине. В частности, переход $B-A$ в сплавах системы $\mathrm{Al}-\mathrm{Mg}$ происходит в интервале $\sim 10^{-3}-10^{-2} \mathrm{~s}^{-1}$ (см. [16-18]). В случае прерывистой ползучести сплава АМг6, как видно из рис. $3, B-A$, а затем переходы $A-B$ происходят в ходе развития отдельного деформационного скачка, когда мгновенная скорость образца проходит через второе критическое значение $\dot{\varepsilon}_{\mathrm{cr} 2} \approx 2 \cdot 10^{-2} \mathrm{~s}^{-1}$.

Рассмотрим теперь зависимость истинного напряжения $\sigma_{t h}$ от истинной деформации $\varepsilon_{t h}$ в ходе развития отдельного деформационного скачка в условиях прерывистой ползучести. Истинное напряжение $\sigma_{t h}$ и истинная деформация $\varepsilon_{t h}$ вычисляются по известным формулам [19]

$$
\begin{aligned}
& \sigma_{t h}=\sigma(1+\varepsilon), \\
& \varepsilon_{t h}=\ln (1+\varepsilon) .
\end{aligned}
$$

Кривые $\sigma_{t h}-\varepsilon_{t h}$ обычно строят для характеризации механических свойств материалов в условиях больших деформаций при испытании с контролируемой постоянной скоростью деформации ( $\dot{\varepsilon}_{0}=$ const) или с постоянной скоростью роста напряжения ( $\dot{\sigma}_{0}=$ const). В случае ползучести обычно строят кривую ползучести, т.е. временную зависимость деформации $\varepsilon(t)$. Однако в условиях прерывистой ползучести может быть полезным построение зависимости $\sigma_{t h}\left(\varepsilon_{t h}\right)$ для измерения приращения истинной деформации $\Delta \delta$, приходящейся на одну деформационную полосу. На рис. 4 представлена кривая $\sigma_{t h}-\varepsilon_{t h}$, полученная исключением времени из временных зависимостей истинного напряжения $\sigma_{t h}(t)$ и истинной

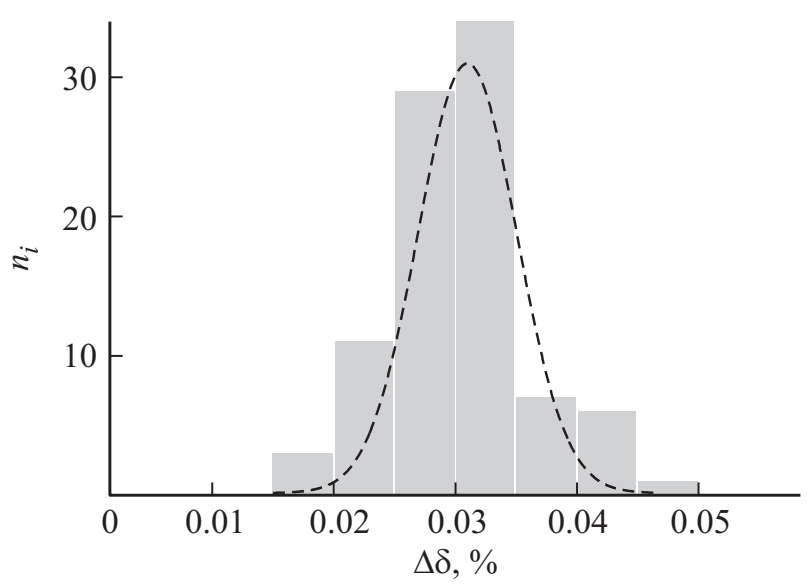

Рис. 5. Гистограмма $n_{i}(\Delta \delta)$ приращений истинной деформации между скачками истинного напряжения. 
деформации $\varepsilon_{t h}(t)$, рассчитанных по формулам (1) и (2) из данных измерений датчиков усилия и деформации, т. е. из экспериментальных кривых $\sigma(t)$ и $\varepsilon(t)$ (рис. 3, кривые 3 и 1 ), в ходе развития деформационного скачка амплитудой $4 \%$.

На рис. 5 показана гистограмма $n_{i}(\Delta \delta)$ приращений $\Delta \delta$ истинной деформации между ближайшими скачками напряжения на участке кривой $\sigma_{t h}-\varepsilon_{t h}$, соответствующем отдельному деформационному скачку. Из рис. 4 и 5 видно, что это приращение деформации почти не меняется в ходе развития макроскопического деформационного скачка и составляет $\Delta \delta=(3.0 \pm 0.57) \cdot 10^{-2} \%$. Учитывая, что каждый скачок напряжения, как обнаружено, связан с зарождением и расширением единственной деформационной полосы, можно заключить, что каждая такая полоса переносит почти одинаковую истинную деформацию; поэтому ее можно рассматривать как „квант“ ${ }^{\text {м }}$ макролокализованной прерывистой деформации в условиях ползучести.

\section{3. Анализ нелинейных колебаний в силовом отклике в ходе развития деформационного скачка}

Описанная выше эволюция пластических неустойчивостей в ходе макроскопической прерывистой ползучести представляет собой яркий пример неравновесного морфогенеза - спонтанного формирования сложных пространственно-временных структур в первоначально однородной неравновесной и нелинейной средах подобно, например, явлению турбулентности, дендритному затвердеванию переохлажденного расплава и т.д. [20]. Как ранее отмечалось, временной ряд $\sigma(t)$, состоящий из многочисленных нелинейных колебаний (в форме повторяющихся скачков напряжения), представляет собой сложный силовой отклик механической системы „образец-испытательная машина“ на спонтанное развитие одиночного макроскопического деформационного скачка в условиях ползучести, когда внешняя сила, действующая на систему, поддерживается постоянной. Этот прерывистый временной ряд, осуществляющий отображение на одну степень свободы эволюции пространственно-временной картины деформационных полос, позволяет, во-первых, контролировать популяции деформационных полос и динамическое поведение различных типов ( $A$ или $B)$ непосредственно в ходе деформирования и, во-вторых, использовать методы статистического анализа временных рядов для исследования явлений самоорганизации и динамического хаоса в деформационном поведении материала.

Статистический анализ. Прежде всего отметим, что несмотря на то что отдельному скачку напряжения соответствует приблизительно одинаковый прирост истинной деформации, амплитуда скачков истинного напряжения распределена в широком интервале от $\sim 0.3$ до $\sim 10 \mathrm{MPa}$, а гистограмма амплитуд скачков напряжения близка к гиперболической форме (рис. 6, $a$ ). В двой-
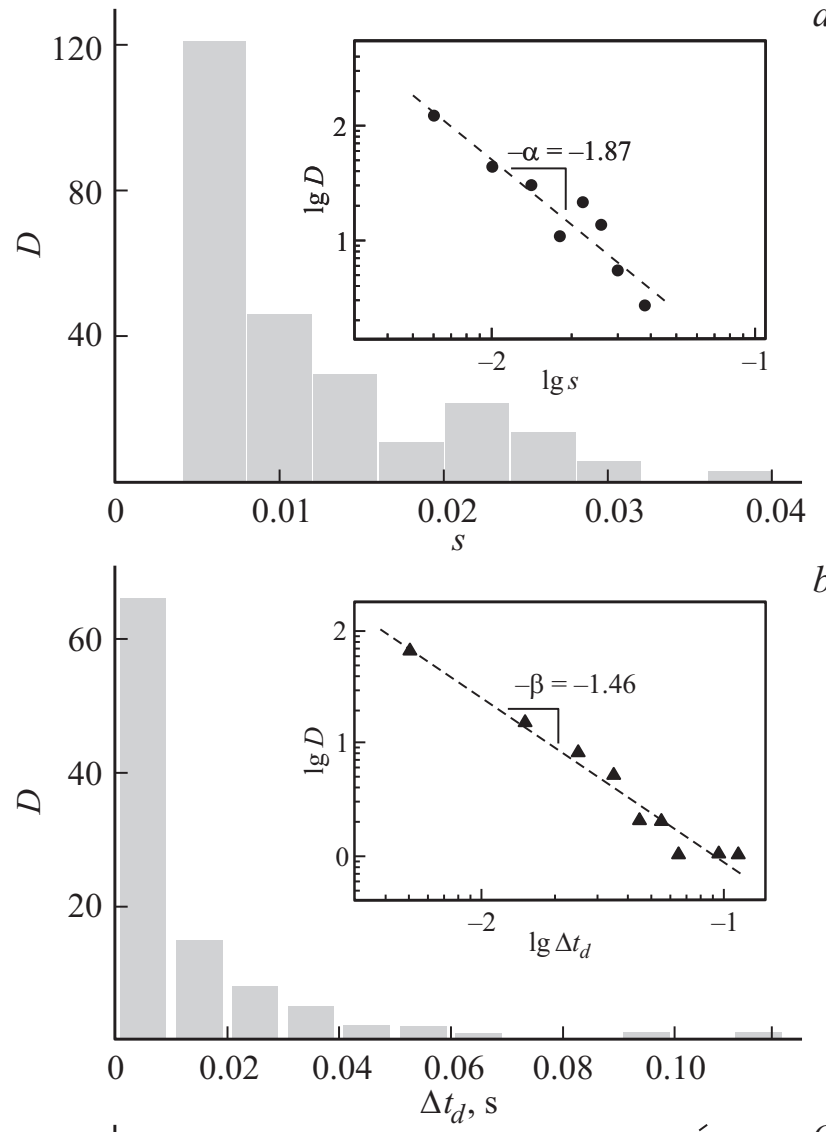

$b$

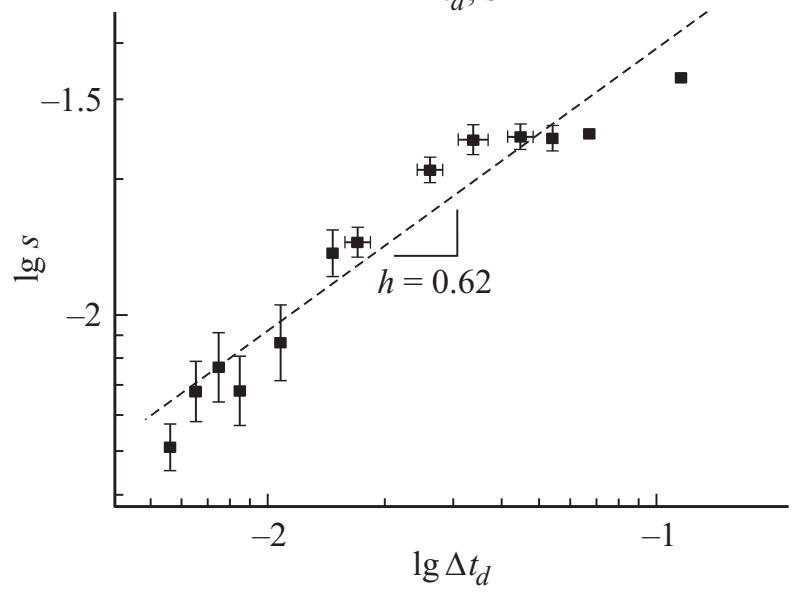

Рис. 6. Результаты статистического анализа скачков напряжения в силовом отклике на развитие деформационного скачка в условиях ползучести. $a$ и $b-$ плотности распределения нормализованной амплитуды $s$ скачка напряжения и его длительности $\Delta t_{d}$ соответственно. Штриховые линии показывают отрицательные склоны этих распределений в двойных логарифмических координатах, которые равны -1.87 и -1.46 для амплитуд скачков напряжения и их длительностей, соответственно. $c-$ зависимость $s$ от $\Delta t_{d}$ в двойных логарифмических координатах. Штриховая прямая линия указывает на степенную зависимость этих величин с показателем степени $h=0.62$.

ных логарифмических координатах статистическая функция распределения $D(s)=N^{-1} d N / d s$ нормализованной амплитуды скачков напряжения $s=\Delta \sigma_{t r} / \sigma_{t r}$ является 
приблизительно линейной с коэффициентом наклона к $s$-оси 1.87 (см. вставку на рис. 6,a). (Здесь $\sigma_{t r}-$ исходный (до скачка) уровень истинного напряжения, $\Delta \sigma_{t r}$ - скачок истинного напряжения, $N$ - общее количество скачков напряжения, $d N-$ количество скачков, амплитуда которых попадает в узкий интервал $(s-\delta s / 2$, $s+\delta s / 2$, см. [21]). Это означает, что функция распределения амплитуд напряжения подчиняется степенному закону

$$
D(s) \sim s^{-\alpha}
$$

с показателем $\alpha=1.87$. Как известно, степенное распределение амплитуд лавин с показателем порядка единицы характерно для землетрясений (закон Гуттенберга-Рихтера [22]) и является парадигмой (точнее, одним из признаков) самоорганизующейся критичности (СОК).

Отметим, что распределение длительности скачков напряжения $\Delta t_{d}$ демонстрирует также скейлинговую форму с показателем $\beta$ :

$$
D\left(\Delta t_{d}\right) \sim \Delta t_{d}^{-\beta} .
$$

В двойных логарифмических координатах нормализованное распределение $D\left(\Delta t_{d}\right)$ показано на рис. $6, b$. Штриховая линия соответствует значению показателя $\beta \approx 1.46$.

Нормализованная амплитуда скачков напряжения соотносится с их продолжительностью степенным законом

$$
s \sim \Delta t_{d}^{h}
$$

На рис. 6, с представлена зависимость $\lg s$ от $\lg \Delta t_{d}$, показывающая значение показателя степени $h=0.7$. Согласно [23], коэффициенты $\alpha, \beta$ и $h$ подчиняются соотношению

$$
\beta=h(\alpha-1)+1 \rightarrow 1.46 \approx 0.7(1.87-1)+1=1.6 .
$$

Скейлинговые законы в уравнениях (3)-(5) и безразмерное соотношение (6) также указывают на состояние самоорганизующейся критичности $[17,23]$.

В названии „СОК“ термин „критичность“ говорит о существовании дальнодействующих корреляций, как в случае фазовых переходов второго рода вблизи критической точки [24]. Таким образом, представленные результаты ясно свидетельствуют о наличии сильных пространственных корреляций пластических неустойчивостей в ходе прерывистой ползучести.

Согласно [25,26], для систем с СОК глобальная динамика, как предполагается, контролируется дальнодействующими корреляциями между большим числом локальных объектов - неравновесных носителей переноса. Из результатов настоящей работы следует, что такими носителями являются расширяющиеся деформационные полосы, т.е. „кванты“ макролокализованной деформации, формирующие пространственно-временные структуры, динамика которых соответствует поведению
ПЛШ (полос типа $A$ и $B$ ), а пространственная корреляция осуществляется за счет эстафетного механизма распространения макролокализованной деформации, когда каждая полоса, за исключением первичной, зарождается на границе предшествующей полосы, где внутренние напряжения превысили некоторое пороговое значение.

Переходы между нелинейными динамическими режсимами и самоорганизующаяся критичность. Переходы между динамическими режимами ПЛШ типов $A, B, C$ обычно изучались в условиях растяжения с постоянной скоростью $\dot{\varepsilon}_{0}=$ const в жестких испытательных машинах [16-18,20,21,27,28]. Традиционное объяснение переходов между этими динамическими режимами при изменении скорости деформирования основано на анализе конкуренции между двумя характерными временами: временем восстановления напряжения $t_{L}$ между последовательными скачками разгрузки и временем пластической релаксации $t_{R}$ внутренних напряжений. Поскольку скоростная чувствительность напряжения течения отрицательна в области эффекта ПЛШ, увеличение скорости деформации приводит, по крайней мере, к трем последствиям: а) уменьшению общего уровня напряжения и, следовательно, увеличению времени релаксации, обусловленному термоактивационным движением дислокаций в неоднородном поле внутренних напряжений в области деформационной полосы; б) уменышению скачка локальной скорости деформации в полосе и в) уменьшению времени восстановления напряжения в полосе скачка напряжения.

При очень низкой скорости деформации время восстановления напряжения очень большое и $t_{L} \gg t_{R}$. Внутренние напряжения полностью срелаксированы, и пространственной корреляции между полосами не наблюдается. Из-за отсутствия пространственной корреляции полосы зарождаются в случайных позициях, в которых среднее напряжение достигает порогового уровня зарождения неустойчивости, что приводит к поведению ПЛШ типа $C$. Ранние модели эффекта ПЛШ пренебрегали пространственной связью и описывали его динамику исключительно в терминах релаксационных колебаний [29] на основе концепции о конкуренции процессов закрепления и открепления дислокаций в поле примесных атомов. Вследствие случайности событий, связанных со скачками разгрузки типа $C$, статистические распределения амплитуд скачков напряжения и интервалов между ними имеют форму, близкую к распределению Пуассона $[16,17]$. С ростом скорости деформации время восстановления напряжения становится меньше, а время пластической релаксации возрастает. Когда эти две величины оказываются одного порядка, внутренние напряжения не полностью релаксируют и способствуют зарождению новых полос в окрестности предыдущих полос, производя прыгающий характер распространения локализованной деформации, связанный с поведением ПЛШ типа $B$.

При высоких скоростях деформации $t_{L} \ll t_{R}$ лишь очень небольшая пластическая релаксация может про- 
исходить в ходе восстановления напряжения, что дает в результате высокую степень пространственной корреляции и квазинепрерывное распространение полос типа $A$. Внутренние напряжения всегда близки к критическому значению начала пластической неустойчивости так, что многие дислокационные ансамбли близки к порогу открепления от примесных атмосфер. В результате дислокационные лавины могут срываться на любом масштабном уровне и в любое время под действием флуктуации внутренних упругих полей напряжений, что приводит к степенным распределениям скачков напряжения и пауз между ними, свободных от выделенного пространственно-временного масштаба. Такая ситуация характерна для систем, демонстрирующих состояние СОК. Режим кроссовера динамических режимов типов $A$ и $B$ ожидается при $t_{L} \sim t_{R}[17]$.

Описанная картина качественно соответствует структуре силового отклика в ходе развития деформационного скачка в условиях прерывистой ползучести сплава АМг6. Основная особенность состоит в том, что в ходе развития деформационного скачка в условиях прерывистой ползучести подвижный захват мягкой испытательной машины на растяжении сначала развивает положительное ускорение $\ddot{\varepsilon}>0$ до точки перегиба, когда $\ddot{\varepsilon}=0$ (точка $P$ на рис. 3), а затем испытывает отрицательное ускорение $\ddot{\varepsilon}<0$ на стадии резкого торможения. Поэтому во временном интервале до точки $P$ мгновенная скорость деформации $\dot{\varepsilon}$ непрерывно возрастает, а затем уменьшается. Когда скорость деформации $\dot{\varepsilon}$ достигает критического значения $\dot{\varepsilon}_{\mathrm{cr} 2} \approx 2 \cdot 10^{-2} \mathrm{~s}^{-1}$ (точка $M$ на рис. 3), время релаксации напряжения $t_{L}$ уменьшается до $\sim 10 \mathrm{~ms}$, в то время как время разгрузки, равное продолжительности первой стадии очень быстрого расширения деформационной полосы (рис. 2), т. е. времени пластической релаксации $t_{R}$, возрастает приблизительно от $1.5 \mathrm{~ms}$ до $8 \mathrm{~ms}$.

В окрестности критической скорости деформации $\dot{\varepsilon}_{\text {cr2 }}$ время разгрузки оказывается сопоставимым с характеристическим временем пластической релаксации $t_{L} \approx t_{R} \approx 8-10 \mathrm{~ms}$ и наблюдается кроссовер динамических режимов $B$ и $A$; а именно скачкообразное распространение локализованной пластической деформации вдоль образца, которое сопровождается скачками напряжения типа $B$ с возрастающей частотой скачков трансформируется в квазинепрерывное распространение полосы типа $A$ со слабыми осцилляциями в силовом отклике $\sigma(t)$. Затем на стадии торможения, когда $\ddot{\varepsilon}<0$, скорость деформации $\dot{\varepsilon}$ непрерывно уменьшается и описанная выше картина „сканируется“ в обратной последовательности: переход от динамического режима $A$ к режиму $B$ происходит вблизи точки $D$, когда скорость деформации упадет до критического значения $\dot{\varepsilon}_{\mathrm{cr} 2} \approx 2 \cdot 10^{-2} \mathrm{~s}^{-1}$ (рис. 3), при котором $t_{L} \approx t_{R}$.

Как отмечалось выше, прямые наблюдения поверхности образца с использованием высокоскоростной видеокамеры показали, что в динамическом режиме типа $B$ каждая полоса зарождается на границе предшествующей полосы. Следовательно, порог неустойчивости достигается на границе полосы, где деформационный градиент максимален. Последний, как представляется, индуцирует упругие напряжения несовместности между пластически деформируемой сдвигом и недеформируемой областями материала. Когда эти напряжения достигают критического значения (например, порогового напряжения открепления дислокаций от примесных атмосфер и/или напряжения коллективного срабатывания источников Франка-Рида или их комбинации), происходит пластическая релаксация этих упругих напряжений вследствие зарождения и расширения новой полосы деформации, что в свою очередь приводит к развитию очередного скачка разгрузки в силовом отклике инерционной испытательной машине на ползучесть.

Скорость деформации всего образца варьируется в относительно большом диапазоне (от $\dot{\varepsilon}_{\mathrm{cr}} \sim 10^{-4} \mathrm{~s}^{-1}$ до около $4 \cdot 10^{-2} \mathrm{~s}^{-1}$ ) в ходе развития отдельного деформационного скачка. Это приводит к скоростному сканированию различных динамических режимов ( $A$ и $B$ ). Результирующее распределение скачков напряжения, как обнаружено, имеет степенной характер, который свидетельствует о тенденции системы к СОК. Этот вывод не является неожиданным, так как основные условия возникновения СОК соблюдаются при прерывистой ползучести: 1) пороговая динамика (критическое напряжение открепления дислокаций от стопоров, и/или коллективное срабатывание источников Франка-Рида и т.д.); 2) очень медленное „внешнее управление“ по отношению к скорости развития внутренних событий (в нашем случае локальные скорости деформации, связанные с деформационной полосой, значительно превышают общую скорость деформации образца, которая предшествует развитию деформационного скачка); 3) наличие двух временных масштабов, быстрого и медленного $\left(t_{L}\right.$ и $\left.t_{R}\right)$; 4) пространственная связь между дефектами, которая, как предполагается, возникает в виде внутренних напряжений, обусловленных геометрическими несовместностями между соседними областями материала и сильно отличающимся уровнем пластической деформации (напряжения градиентной пластичности).

Таким образом, нелинейная дислокационная динамика в ходе макроскопической прерывистой ползучести сплава системы $\mathrm{Al}-\mathrm{Mg}$ представляет новый пример состояния самоорганизующейся критичности. Отметим, что степенная статистика наблюдалась для скачков напряжения при низкотемпературной скачкообразной деформации ниобия в жесткой испытательной машине [30], для дискретных событий акустической эмиссии поликристаллического сплава $\mathrm{Al}-\mathrm{Mg}$, деформируемого с постоянной скоростью $\dot{\varepsilon}_{0}=$ const в условиях эффекта ПЛШ [31,32], а также для амплитуд сигналов АЭ при макроскопически непрерывной ползучести монокристаллов льда [33] и сигналов электромагнитной эмиссии при одноосном сжатии льда, связанной с динамикой дислокационных лавин и трещин при активном нагружении в мягкой машине [34]. 
В настоящей работе с помощью in situ экспериментов установлен „мезоскопический “ механизм прерывистой ползучести, состоящий в спонтанном формировании в течение десятых долей секунды сложной пространственно-временной структуры деформационных полос, которая вызывает развитие макроступени на кривой ползучести амплитудой несколько, до $\sim 10 \%$. Микроскопическая природа прерывистой ползучести остается открытым вопросом, требующим дальнейших исследований.

\section{Заключение}

Основные результаты, полученные в работе, состоят в следующем.

1. Прерывистая ползучесть проявляет пороговую динамику: деформационный скачок стартует, когда скорость предшествующей непрерывной ползучести достигает некоторого критического значения. Установлены эмпирические условия возникновения деформационного скачка в сплаве АМг6 при комнатной температуре: до начала скачка скорость ползучести меняется от постоянной (подобно второй стадии ползучести) до растущей по степенному закону (подобно третьей стадии ползучести) до критического значения $\dot{\varepsilon}_{\mathrm{cr} 1} \sim 10^{-4} \mathrm{c}^{-1}$.

2. Каждый скачок напряжения в структуре силового отклика коррелирует с одной мезоскопической деформационной полосой, которая представляет собой расширяющуюся шейку, наклоненную к оси образца на угол около $60^{\circ}$. Эволюция полосы характеризуется двумя последовательными стадиями - быстрой и медленной. На медленной стадии скорость границ полосы постепенно уменьшается, и когда она упадет до нижнего предела (около $\sim 1 \mathrm{~mm} / \mathrm{s}$ ), границы полосы генерируют вторичные полосы, границы вторичных полос генерируют полосы третьего поколения и т.д. В результате эстафетной передачи пластической деформации от одной мезополосы к другой, когда каждая полоса, за исключением первичной, зарождается на границе предыдущей полосы, макролокализованная деформация распространяется прыжками вдоль направления растяжения образца подобно макрополосе ПЛШ типа $B$.

3. В ходе развития деформационного скачка скорость деформации меняется в интервале $\sim 10^{-4}-4 \cdot 10^{-2} \mathrm{~s}^{-1}$ и происходит переход между динамическими режимами ПЛШ типов $A$ и $B$, когда скорость деформации проходит через второе критическое значение $\dot{\varepsilon}_{\mathrm{cr} 2} \approx 2 \cdot 10^{-2} \mathrm{~s}^{-1}$. Эти режимы характеризуются различными уровнями регулярности скачков напряжения в силовом отклике.

4. Установлено, что временная структура силового отклика на развитие макроскопической ступени на кривой ползучести демонстрирует тенденцию к состоянию самоорганизующейся критичности, представляющей явный признак нелинейного поведения материала в условиях прерывистой ползучести.

Экспериментальная часть работы выполнена при поддержке гранта РФФИ (проект № 16-08-00773), а статистический анализ поученных результатов и их обсуждение - при поддержке Российского научного фонда (проект № 15-12-00035).

\section{Список литературы}

[1] Estrin Y., Kubin L.P. Continuum models for materials with microstructures / Ed. by H.-B. Muhlhaus. NY.: Wiley \& Sons, 1995. P. 395-453.

[2] Kubin L.P., Fressengeas C., Ananthakrishna G. In: Nabarro F.R.N., Duesbery M.S., ed. Dislocations in solids. Amsterdam: Elsevier. 2002. P. 101-192.

[3] Rizzi E., Hahner P. // Int. Journ. Plasticity. 2004. Vol. 20. P. $121-165$.

[4] Белл Джс.Ф. Экспериментальные основы механики деформируемых твердых тел. Ч. 2. М.: Наука, 1984. 432 с.

[5] Kubin L.P., Estrin Y. // Acta Metall. 1985. Vol. 33 (3). P. $397-$ 407.

[6] Шибков А.А., Золотов А.Е. // Письма в ЖЭТФ. 2009. Т. 90. Вып. 5. С. 412-417.

[7] Andrade E.N. da C. // Proc. Roy. Soc. 1910. Vol. 84. P. 1-12.

[8] Klueh R.L., King J.F. // Scr. Metall. 1979. Vol. 13. P. 205-209.

[9] Klueh R.L., King J.F. // J. Nuclear Mater. 1981. Vol. 98. P. 173-189.

[10] Ananthakrishna G., Sahoo D. // J. Phys. D: Appl. Phys. 1981. Vol. 14. P. 2081-2090.

[11] Shibkov A.A., Denisov A.A., Zheltov M.A., Zolotov A.E., Gasanov M.F. // Mater. Sci. Eng. A. 2014. Vol. 610. P. 338 343.

[12] Шибков А.А., Золотов А.Е., Желтов М.А. // Известия РАН. Серия физическая. 2012. Т. 76. Вып. 1. С. 97-107.

[13] Шибков А.А., Золотов А.Е., Желтов М.А., Денисов А.А., Гасанов М.Ф. // ЖТФ. 2014. Т. 84. Вып. 4. С. 40-46.

[14] Шибков А.А., Золотов А.Е., Желтов М.А., Гасанов М.Ф., Денисов А.А. // ФТТ. 2014. Т. 56. Вып. 5. С. 848-855.

[15] Шибков А.А., Мазилкин А.А., Протасова С.Г., Михлик Д.В., Золотов А.Е., Желтов М.А., Шуклинов А.В. // Деформация и разрушение материалов. 2008. Вып. 5. C. 24-32.

[16] Bharathi M.S., Ananthakrishna G., Fressengeas C., Kubin L.P., Lebyodkin M. // Phys. Rev. Lett. 2001. Vol. 87. P. 165508.

[17] Bharathi M.S., Lebedkin M., Ananthakrishna G., Fressengeas C., Kubin L.P. // Acta Mater. 2002. Vol. 50. P. 2813-2824.

[18] Chatterjee A., Sarkar A., Barat P., Mukherjee P., Gayathri N. // Mater. Sci. Eng. A. 2009. Vol. 508. P. 156160.

[19] Новиков И.И., Портной В.К. // Сверхпластичность сплавов с ультрамелким зерном. М.: Металлургия, 1981. 168 с.

[20] Kessler D.A., Koplik J., Levine A. // Adv. Phys. 1988. Vol. 37. P. 255-339.

[21] Lebedkina T.A., Lebyodkin M.A. // Acta Mater. 2008. Vol. 56. P. 5567-5574.

[22] Gutenberg B., Richter C.F. // Ann. di Geophisica. 1956. Vol. 9. P. 1-15.

[23] Kertesz J., Kiss B.L. // J. Phys. A: Math. Gen. 1990. Vol. 20. P. L433-L440.

[24] Лифиии, Е.М., Питаевский Л.П. // Физическая кинетика. М.: Наука, 1979. 528 с.

[25] Bak P., Tang C., Wiessenfeld K. // Phys. Rev. A. 1988. Vol. 38. P. 364-374.

[26] Jensen H.J. Self-organized criticality. Cambridge: Cambridge University Press, 1998.

[27] Shashkov I.V., Lebyodkin M.A., Lebedkina T.A. // Acta Mater. 2012. Vol. 60. P. 6842-6850. 
[28] Lebyodkin M., Brechet Y., Estrin Y., Kubin L.P. // Phys. Rev. Lett. 1995. Vol. 74. P. 4758-4761.

[29] Penning P. // Acta Metall. 1972. Vol. 20. P. 1169-1175.

[30] Бобров В.С., Зайцев С.И., Лебедкин М.А. // ФТТ. 1990. T. 32. Вып. 10. С. 3060-3065.

[31] Lebyodkin M., Brechet Y., Estrin Y., Kubin L.P. // Phys. Rev. Lett. 1995. Vol. 74. P. 4758-4761.

[32] Lebyodkin M.A., Estrin Y. // Acta Mater. 2005. Vol. 52. P. 3403-3413.

[33] Weiss J., Grasso J.-R. // J. Phys. Chem. B. 1997. Vol. 101. P. 6113-6117.

[34] Шибков А.А., Казаков А.А. // Кристаллография. 2009. Т. 54. Вып. 2. С. 323-330. 